\title{
Eventraciones. Reconstitución plástica de la línea alba mediante doble sutura invaginante isotensional (DSII)*
}

\author{
Drs. ALBERTO ACEVEDO F. ${ }^{1}$, JUAN LOMBARDI S. ${ }^{1}$, JORGE LEÓN S. ${ }^{1}$, \\ JACQUELINE LÓPEZ R. ${ }^{1}$, ERNESTO SCHULTZ F. ${ }^{1}$, EU. VERÓNICA DELLEPIANE S. ${ }^{1}$
}

1 Programa de Cirugía Mayor Ambulatoria (CMA) del Centro de Referencia de Salud (CRS) Cordillera Oriente, Facultad de Medicina, Universidad de Chile. Santiago, Chile.

Abstract

\section{Reconstruction of midline incisional hernias using a double isotensional suture}

Background: Incisional hernias of the midline are frequently multisacular and its treatment without a mesh is followed by a high rate of recurrences. Aim: To report a new technique for the treatment of midline incisional hernias, performing an isotensional suture of the midline with $\mathrm{Vycril}^{\circledR} 2-0$, followed by a double invaginant suture of the rectus aponeurosis with Ethybond ${ }^{\circledR} 2-0$. Material and Methods: Observational prospective study of 90 women and 26 men, aged $54 \pm 13$ and $59 \pm 13$ years respectively, with a hernia sac smaller than $15 \mathrm{~cm}$ and with an aponeurotic defect smaller than $3 \mathrm{~cm}$, operated between January 2002 and December 2005. Results: Surgery was performed under local anesthesia on an ambulatory basis. The surgical procedure consisted in the excision of a skin lozenge which included the scar, dissection and reduction of all sacs and isotensional suture of the midline with Vycryl $\# 1^{\circledR}$. This suture was followed by a double invaginating suture of the rectus sheath in the midline with Ethylon ${ }^{\circledR} 0$. Five superficial infections and two hematomas were observed. Seven recurrences $(7 \%)$ in 99 patients $(85 \%$ of the sample), were found during a mean follow up of 4.6 years (range 2-6). Conclusions: The double invaginating isotensional suture (DIIS) is an acceptable alternative for the treatment of small and middle size incisional hernias of the midline.

Key words: Incisional hernia, isotensional suture, hernia repair.

\section{Resumen}

Las eventrorrafias sin malla de la línea media se asocian con una elevada tasa de recidivas. En esta publicación damos cuenta de una técnica fascial utilizable en eventraciones pequeñas y medianas de la línea media que hemos llamado doble sutura invaginante isotensional (DSII). El estudio observacional descriptivo es una serie clínica constituida por 90 mujeres con una edad promedio de $54 \pm 12,9$ años y 26 varones con una edad promedio de $59 \pm 13,7$ años, intervenidos entre el $1^{\circ}$ de Enero de 2002 y el 31 de Diciembre de 2005. En 14 casos se trató de una eventración recidivada. Se intervinieron pacientes con un

*Recibido el 11 de Octubre de 2008 y aceptado para publicación el 22 de Marzo de 2009.

Correspondencia: Dr. Alberto Acevedo F.

Las Torres 5100, Santiago, Chile.

E-mail: aacevedof@gmail.com 
saco de hasta $15 \mathrm{~cm}$, único o múltiple, con un anillo inferior a $3 \mathrm{~cm}$, con una edad fisiológica inferior a los 70 años y ASA 1 y 2 . La obesidad y la diabetes mellitus no constituyeron una contraindicación. La operación se efectuó con anestesia local, en forma ambulatoria en 98 y con anestesia raquídea en 18 casos. La técnica incluyó resección de un losanjo de piel, manejo de los sacos y rrafia mediante sutura de la línea alba con Vycryl® 2-0, seguida de doble sutura invaginante del plano aponeurótico con Ethybond ${ }^{\circledR} 0$. Apreciamos 5 infecciones y 2 hematomas de la herida. Un seguimiento promedio de 4,6 (2 - 6) años se efectuó en 99 pacientes $(85 \%)$ de la muestra, apreciándose 7 recidivas (7\%). La DSII es una técnica alternativa en las eventraciones pequeñas y medianas de la línea media.

Palabras clave: Eventración de la línea media, anestesia local, cirugía ambulatoria.

\section{Introducción}

Desde su inauguración de la unidad, en Agosto del año 1997, iniciamos un programa de cirugía ambulatoria de las hernias dirigido a los pacientes con patología de la pared abdominal, provenientes de los 5 consultorios de las comunas de Macul y Peñalolén con una población asignada de 298.760 habitantes adscritos a la Atención Primaria de Salud (APS). Desde esa fecha han sido derivados al consultorio externo 2.312 pacientes portadores de patología de pared, constituyendo las eventraciones la tercera patología más frecuente con un $11,7 \%{ }^{1}$.

Habiendo comprobado que las eventraciones de la línea media son frecuentemente multisaculares y que comprometen a la cicatriz en toda su extensión $^{2}$, hemos desarrollado una técnica basada en tres principios: Explorar toda la cicatriz operatoria, evitar la tensión exagerada sobre la sutura, y reconstituir la línea alba, acercando los músculos rectos a la línea media. A la reconstitución de la línea alba la denominamos doble sutura invaginante isotensional (DSII). La Tabla 1 permite apreciar las técnicas utilizadas en el período de tiempo que abarca el presente estudio en el Programa CMA del CRS Cordillera ${ }^{3}$, agregando 18 pacientes que durante dicho tiempo fueron operados en el Hospital del Salvador utilizando la técnica de DSII. El objetivo de este estudio es dar a conocer nuestros resultados a largo plazo con esta técnica en una serie clínica de pacientes intervenidos en un período de 4 años.

Tabla 1. Tipo de rrafia en las eventraciones de la línea media

\begin{tabular}{lrr}
\hline Eventrorrafias & $\mathbf{n}$ & $\mathbf{\%}$ \\
\hline DSII* & 116 & 87,7 \\
Malla preperitoneal & 23 & 16,3 \\
Sutura simple & 9 & 6,0 \\
Total & 148 & 100,0 \\
\hline
\end{tabular}

*14 rrafias efectuadas en el Hospital del Salvador.

\section{Material y Método}

El estudio observacional descriptivo incluye 147 casos. De éstos, 127 fueron eventraciones de la línea media y 98 fueron tratadas con la técnica de DSII. Hemos agregado 18 pacientes intervenidos con esta técnica, en este mismo período de tiempo, en el Servicio de Cirugía del Hospital del Salvador, completando una serie clínica de 116 casos.

Criterios de inclusión: ASA 1 ó 2, disponer de una vivienda y condiciones familiares estables, tener una condición neuropsiquiátrica compatible, tener una eventración con uno o varios sacos de diámetro inferior a los $15 \mathrm{~cm}$ y un anillo eventral de un diámetro transversal de menos de $3 \mathrm{~cm}$. La obesidad no constituyó una contraindicación para el tratamiento.

Usamos una premedicación consistente en $2 \mathrm{mg}$ de Dormonid ${ }^{\circledR}$ y $2 \mathrm{mg}$ de Petidina ${ }^{\circledR}$ administrados en la vena, al momento de iniciar la intervención. Esta dosis se repitió en caso necesario. Se efectuó quimioprofilaxis con Cefazolina ${ }^{\circledR} 1$ gr diluido en 10 $\mathrm{ml}$ de solución que se administró igualmente por vía intravenosa inmediatamente antes de la cirugía.

La anestesia local, monitorizada, se efectuó infiltrando sucesivamente los planos: dermis, tejido celular subcutáneo y plano subaponeurótico. Se utilizaron $300 \mathrm{ml}$ de Lidocaína ${ }^{\circledR}$ al $0,5 \%$, alcalinizada y con adición de Efedrina. En el plano subaponeurótico, efectuamos un bloqueo de los nervios intercostales en su trayecto dentro de la vaina de los rectos. Con este propósito el anestésico se inyecta dentro de la vaina de los rectos en un volumen de $2 \mathrm{ml}$ cada $2 \mathrm{~cm}$ en toda la longitud del defecto.

Los 18 pacientes intervenidos en el Hospital del Salvador recibieron anestesia raquídea. En 16 de ellos se utilizó drenaje con succión a presión negativa $\left(\operatorname{Redon}^{\circledR}\right)$ y permanecieron hospitalizados hasta su retiro.

Técnica quirúrgica. Extirpamos la cicatriz incluida en un losanjo de piel de la longitud y ancho adecuados, exponiendo la aponeurosis sólo lo necesario para exponer el o los anillos eventuales y realizar la doble sutura invaginante, habitualmente 


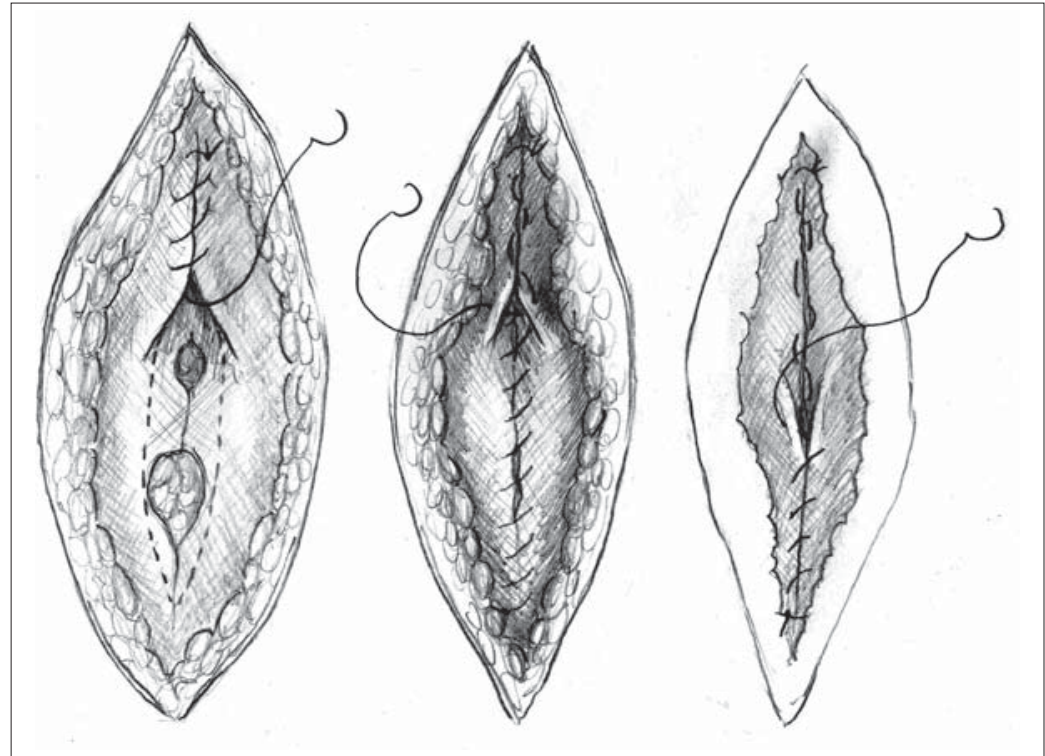

Figura 1. Esquema de la doble sutura isotensional de la línea blanca. Izq: Sutura isotensional; Medio: Primer plano de la doble sutura invaginante; Der: Segundo plano.
$6 \mathrm{~cm}$. Luego de la disección y reducción de los sacos, efectuamos una sutura simple, corrida, con Vicryl ${ }^{\circledR} 2-0$, que ocluye los anillos y constituye una primera sutura invaginante de la línea media, que consigue que la tensión se distribuya en forma homogénea en toda la longitud de la sutura. Esta sutura, que llamamos isotensional, la efectuamos procurando mantener una relación longitud del hilo de sutura/longitud de la herida de 4, según recomienda Israelsson ${ }^{4}$. Este plano fue seguido de una doble sutura invaginante de la vaina de los rectos con Ethybond ${ }^{\circledR} 1$ (Figura 1). Inicialmente utilizamos un drenaje de Penrose que hemos sustituido en los últimos 86 pacientes por la fijación del tejido celular subcutáneo al plano aponeurótico mediante puntos separados de Vycril ${ }^{\mathrm{R}} 1$ (sutura anclada del celular subcutáneo) $)^{5}$.

Previo al alta un miembro del equipo evaluó el dolor y la satisfacción experimentados por el paciente durante el acto quirúrgico, mediante EVA de 10 puntos. Esto se repitió en el primer control efectuado al día siguiente de la intervención.

Los controles postoperatorios se efectuaron a las 24 horas, al séptimo y al trigésimo día postoperatorio, mediante un protocolo preestablecido. La información obtenida se introdujo en la base de datos del proyecto de pared abdominal elaborado mediante el software de cálculo epidemiológico Epi Info 2000.

El seguimiento a largo plazo se efectuó en forma personal por un miembro del equipo, con un período de observación promedio de 4,6 (2-6) años. El examen se realizó en posición de pie y en decúbito supino elevando la cabeza y ambas extremidades inferiores para aumentar la presión intra-abdominal como la recomienda Israelsson ${ }^{4}$.

La probabilidad estadística se determinó mediante la prueba de $\chi^{2}$.

\section{Resultados}

Los antecedentes patológicos de la muestra pueden apreciarse en la Tabla 2. La tasa de obesidad en las mujeres fue significativamente mayor en las mujeres, con un $60,2 \%$ que en los varones, con un $28 \%(\mathrm{p}<0,005)$.

Tabla 2. Antecedentes mórbidos de los pacientes sometidos a una DSII

\begin{tabular}{lrr}
\hline Antecedentes & n & \% \\
\hline Sin antecedentes & 56 & 48,3 \\
Obesidad (IMC > 30) & 55 & 47,4 \\
Obesidad mórbida (IMC > 40) & 5 & 4,3 \\
Hipertensión (HT) & 35 & 30,2 \\
Diabetes mellitus (DM) & 4 & 3,6 \\
HT + DM & 3 & 2,8 \\
Asma bronquial & 5 & 4,3 \\
Otros & 1 & 0,9 \\
Total & 116 & \\
\hline
\end{tabular}


La hipertensión arterial y la diabetes mellitus fueron los antecedentes mórbidos más frecuentes, similar en ambos sexos.

La eventrorrafia ambulatoria con anestesia local pudo efectuarse sin mayores incidentes en la totalidad de los pacientes intervenidos en el CRS Cordillera. En 3 oportunidades la separación de los bordes aponeuróticos demostró ser mayor que lo establecido en el examen de ingreso y ello hizo necesaria la colocación de una malla.

La localización de las eventrorrafias se aprecia en la Tabla 3. Estas fueron unisaculares en 32\% de los casos y multisaculares en el resto. Una hernia umbilical se asoció en el 33\% y una diástasis en el $31 \%$ de los pacientes.

La duración del procedimiento fue 66 minutos (45-141); la extensión mayor de la incisión hizo necesaria la administración de sedantes y analgésicos, lo que se fue imponiendo en la medida que adquiríamos experiencia en la realización de la técnica con anestesia local. El dolor operatorio fue calificado con 2,9 (1-8) puntos de la EVA, refiriendo el $88 \%$ de los pacientes un dolor inferior a 4 . La satisfacción del usuario se calificó con un puntaje de 8,7 (6-10) con un $98 \%$ de los enfermos con un puntaje superior a 7 .

En el control efectuado a la semana, comprobamos la persistencia del dolor con una puntuación promedio de 4 (1-7), la que es mayor a la experimentada durante el acto quirúrgico. El dolor se localizó más en los flancos que en la herida operatoria misma y no dificultó el reintegro de los enfermos a sus actividades habituales. En este período comprobamos en el $28 \%$ de los pacientes náuseas leves y transitorias que no impidieron la ingesta alimenticia. No observamos cefalea, problemas urinarios, vasculares o digestivos. La satisfacción al séptimo día del postoperatorio fue de 6 (3-10) como consecuencia del dolor referido.

Se detectaron 5 infecciones de la herida y 1 hematoma que requirieron drenaje y fueron manejados ambulatoriamente. Un segundo hematoma de mayor tamaño requirió hospitalización y drenaje quirúrgico. En esta serie clínica no hemos observado seromas.

Tabla 3. Localización de la eventración

\begin{tabular}{lrr}
\hline Localización & n & \% \\
\hline Xifoumbilical & 60 & 50,0 \\
Umbilicopubiana & 44 & 38,5 \\
Xifopubiana & 8 & 7,3 \\
Paraumbilical & 4 & 4,2 \\
Total & 116 & 100,0 \\
\hline
\end{tabular}

El seguimiento fue posible en 99 pacientes $(85,4 \%)$, comprobándose la defunción de 2 pacientes por causas desvinculadas de la herniorrafia. Los 15 pacientes restantes habían cambiado de domicilio y no fueron ubicables. El examen efectuado en los 99 pacientes se realizó con los criterios estrictos de Israelsson y permitió pesquisar 7 recidivas (7\%) con un promedio de seguimiento de 4,6 (2-6) años. No observamos fístulas $u$ otras anormalidades de la cicatrización.

\section{Discusión}

Hemos encontrado escasas publicaciones que den cuenta del uso de anestesia local en el tratamiento quirúrgico de las eventraciones. Privitera y $\operatorname{cols}^{6}$, utilizan anestesia local y "one day surgery" para tratar eventraciones pequeñas y Schumpelick ${ }^{7}$ y Kingsnorth y LeBlanc ${ }^{8}$, señalan que la anestesia local puede usarse en eventraciones de pequeño tamaño $(<4 \mathrm{~cm})$, pero no dan cuenta de su experiencia personal ni aclaran qué llaman eventración pequeña.

En el equipo de cirugía mayor ambulatoria del CRS Cordillera, el uso de anestesia local para un número creciente de afecciones quirúrgicas de la pared abdominal es la consecuencia natural de la mayor destreza en la técnica y de una permanente inquietud por ampliar sus posibilidades en beneficio de los enfermos.

El uso de volúmenes mayores de anestésico más diluido y la infiltración anestésica del músculo recto dentro de su vaina posibilitaron el desarrollo de la técnica quirúrgica que acá analizamos y que beneficia a eventraciones pequeñas y medianas con una separación de los bordes del músculo recto de hasta $3 \mathrm{~cm}$.

El uso exitoso de esta técnica en pacientes obesos y obesos mórbidos, que constituyen el $50 \%$ de esta serie clínica, es especialmente destacable tanto porque el uso de la anestesia local disminuye los riesgos anestésicos, como porque la técnica propuesta se ejecuta sin mayor problema en ellos.

Schumpelick $^{1}$, Barroetaveña y Herzage ${ }^{9}$, consideran pequeñas a las eventraciones con un anillo inferior a $4 \mathrm{~cm}$, pero en nuestra experiencia los anillos de $4 \mathrm{~cm}$ no permiten un afrontamiento sin tensión de los bordes en la línea media en la mayoría de los casos y por ello hemos puesto el límite de 3 $\mathrm{cm}$ para efectuar la sutura que llamamos isotensional. Cuando el anillo es de $4 \mathrm{~cm}$ o más, empleamos una malla "sublay" instalada con la técnica de Rives $^{10} \mathrm{o}$ la técnica "onlay" de Chevrel ${ }^{11}$. En la práctica, en tres pacientes inicialmente agendados para una DSII por presentar al examen de ingreso un anillo de hasta $3 \mathrm{~cm}$, se debió modificar la técnica 
por comprobarse un diámetro mayor durante la intervención; se instaló una malla "sublay". En otros 3 pacientes con una separación del borde aponeurótico mayor de $3 \mathrm{~cm}$ pudimos emplear la DSII ya que los bordes se aproximaron a la línea media sin tensión. La Tabla 4 reproduce nuestro criterio actual para clasificar las eventraciones de la línea media, basado en la indicación terapéutica.

El dolor operatorio y la satisfacción del usuario obtenidos con esta técnica son comparables a los obtenidos en los pacientes sometidos a herniorrafias inguinales recidivadas, inguinoescrotales o de gran tamaño ${ }^{1}$.

Un problema aún por resolver es el control del dolor en los primeros días del postoperatorio en los pacientes intervenidos de una eventrorrafia. Éste se localiza principalmente en ambos hipocondrios y flancos y se exacerba con los movimientos, la respiración profunda y la tos. Es necesario que el paciente y sus familiares sean informados al respecto y que se prescriban analgésicos más potentes. En el postoperatorio precoz la satisfacción del usurario medido con EVA bajó por este motivo a un promedio de 6 (3-10).

Puestos a establecer la proporción de recidivas en los estudios de la literatura, hemos podido comprobar una falta de uniformidad para establecer la presencia de una recidiva y del momento en que ésta debe buscarse ${ }^{12-14}$. Hemos decidido, para los efectos de este estudio usar el criterio propuesto por Israelsson ${ }^{4}$. Para este autor un examen cuidadoso de la cicatriz efectuado personalmente por un miembro del equipo quirúrgico, de pie y en decúbito, asociado a maniobras para aumentar la presión intra-abdominal, debe poner de manifiesto prácticamente el $100 \%$ de las eventraciones. No sólo debe buscarse el bulto de una eventración, sino que también debilidades o separaciones anormales de las capas aponeuróticas suturadas.

En 7 pacientes pudimos comprobar una recidiva, lo que constituyó el 7\% de la muestra, cifra extremadamente favorable y comparable con los mejores resultados obtenidos con otras técnicas de sutura simple y aún a los obtenidos tras la colocación de mallas ${ }^{14-17}$. Así se describen recidivas del $27 \%$ con la técnica de Welti Eudel ${ }^{18}$, superiores al 20\% con la de Mayo $^{16}$ y de $18 \%$ con diversas técnicas de sutura simple ${ }^{13}$. En un meticuloso estudio, Langer $^{12}$ da cuenta de una recidiva del $37 \%$ con la técnica de Mayo y describe el uso decreciente de esta técnica en el Departamento de Cirugía General de la clínica quirúrgica de la Universidad de Gotinga en Alemania, para ser reemplazada por técnicas con malla de Polipropileno.

Si bien el uso de mallas de Polipropileno ha disminuido la frecuencia de las recidivas éstas consti- tuyen aún la más frecuente de las complicaciones tardías. De especial interés nos parece el estudio prospectivo, randomizado de que da cuenta Luijedijk $^{13,14}$, por tratarse de eventraciones de tamaño pequeño y mediano en las cuales la recidiva de la reparación con malla fue de $24 \%$. Algo mejores fueron los resultados comunicados por Sauerland y cols ${ }^{15}$, que dan cuenta de una recidiva del $5 \%$ en las eventraciones operadas con malla.

Destacamos la publicación de Israelsson y cols ${ }^{17}$, que representa un estudio que abarcó 40 hospitales de Suecia con un seguimiento de 2 años. En las hernias con un anillo inferior a los $3 \mathrm{~cm}$ la recurrencia fue de $27 \%$ al emplear una sutura simple y de $10 \%$ tras el empleo de una malla de Prolene "onlay".

Similares resultados son comunicados por Korenkov $^{20}$, de la Universidad de Colonia que destaca recidivas superiores al $10 \%$ en las hernias de tamaño pequeño o mediano, tanto para la sutura simple como para la herniorrafia con malla.

Se ha destacado la desventaja que representa la mayor frecuencia de hematomas e infecciones y seromas observadas con el uso de mallas, que obliga a acciones quirúrgicas que prolongan la hospitalización, el tratamiento y los $\operatorname{costos}^{10,13,19,20}$

En las eventraciones de tipo grande y muy grande (Tabla 4), las mallas son una necesidad, sin embargo, debemos destacar que el $69 \%$ de los pacientes que consultaron en nuestro consultorio externo por una eventración el anillo fue de $3 \mathrm{~cm}$ o menos. En estos pacientes la DSII tiene ventajas tanto por la baja frecuencia de complicaciones postoperatorias como por la baja tasa de recidivas. A estas dos ventajas debe agregarse la curva de aprendizaje corta y que puede ser realizada en forma ambulatoria con anestesia local aun en establecimientos hospitalarios periféricos y en aquellos diseñados para la realización de procedimientos de baja complejidad.

Conviene destacar la elevada frecuencia con que una diástasis de los rectos o una hernia umbilical acompañan a las eventraciones de la línea media. Todo ello está poniendo en evidencia una debilidad de la línea alba que hace necesario su reparación integral. La técnica que proponemos consigue este objetivo por varias razones:

1. Exploramos toda la cicatriz operatoria exponiendo la línea alba en toda su longitud y el ombligo.

2. Reparamos la línea alba en toda su longitud, primero por la sutura que hemos llamado isotensional y segundo por la doble sutura invaginante que aproxima los bordes mediales de los rectos.

3. En este proceso se desprende la cicatriz umbilical y se involucra la hernia o la cicatriz umbilical en la DSII. Por todo ello, hablamos de una reconstitución plástica de la línea alba. 
Tabla 4. Clasificación de las eventraciones crónicas de la línea media

\begin{tabular}{|c|c|c|c|c|}
\hline Tipo & Anillo & $\begin{array}{c}\text { Objetivación } \\
\text { (Diámetro del anillo) }\end{array}$ & Anestesia & Tratamiento propuesto \\
\hline Pequeña & $<1,5 \mathrm{~cm}$ & Dedo índice & Local & Sutura simple, DSII \\
\hline Mediana & $<3 \mathrm{~cm}$ & Dos dedos & Local, espinal, peridural, otra & $\begin{array}{l}\text { Mayo longitudinal, DSII, } \\
\text { Welti Eudel, Cardif, otras }\end{array}$ \\
\hline Grande & $4-7 \mathrm{~cm}$ & Cuatro dedos & $\begin{array}{l}\text { Peridural continua asociada } \\
\text { a general }\end{array}$ & $\begin{array}{l}\text { Malla sublay, Rives, } \\
\text { Malla onlay, Cheverel, otras }\end{array}$ \\
\hline Muy grande & $>7 \mathrm{~cm}$ & $>$ cuatro dedos & $\begin{array}{l}\text { Peridural continua asociada } \\
\text { a general }\end{array}$ & $\begin{array}{l}\text { Plastías múculoapo-neuróticas } \\
\text { (Albanese o otras) }\end{array}$ \\
\hline Complicada & Variable & Variable & Peridural continua & Variable \\
\hline
\end{tabular}

\section{Referencias}

1. Acevedo A, Gallego A, Cirugía mayor ambulatoria de las hernias. Experiencia de 5 años en el CRS Cordillera Oriente de la ciudad de Santiago. Rev Chil Cir 2004; 56: $166-171$.

2. Acevedo A. Multisacularidad de las eventraciones de la Línea media. Cuaderno de Resúmenes LXXVII Congreso Chileno de Cirugía, Pucón, 2004.

3. Acevedo A, Viterbo A, Bravo J, Dellepiane V. Eventraciones, cirugía ambulatoria con anestesia local. Rev Chil Cir 2006; 58: 354-358.

4. Israelsson LA, Johnson T. Incisional hernias after midline laparotomy. A prospective study. Eur J Surg 1996; 162: 125-129.

5. Acevedo A, Viterbo A, Cápona R, Dellepiane V. Prescindencia del drenaje en el eventrorrafia ambulatoria. Rev Chil Cir 2007; Supl: 124.

6. Privitera A, Brancato G, Donati M, Gandolfo L, Cavallaro G, Donati A. Plug technique for small incisional hernia. Ann Ital Chir 2003; 74: 701-704.

7. Schumpelick V, Klosterhalfen V, Müller M, Ling U. Minimierte Polipropilen-Netze zur preperitonealen Netzplastik der Narbenhernie. Chirurg 1999; 70: 422430.

8. Kingsnorth A, LeBlanc KA. Managment of abdominal hernias (third edition). Arnold publishers, 2003, pp 262-293.

9. Barroetaveña J, Herszage L, Tbaudin H, Barroetaveña JL, Ahualli CE. Cirugía de las eventraciones. Editorial el Ateneo, Buenos Aires 1988, pp 104.

10. Flemont JB, Aviasse C, Palot JP. Biomaterials, principles of implantation. En Schumplick V, Kingsnorth A. eds. Incisional hernia. New York, Springer Verlag 1999; pp 376.

11. Chevrel JP. Treatment of incisional hernias by an overlapping herniorraphy an onlay prosthetic implant.
En Bendavid R, Abrahamson J, Arregui ME, Falament JB, Phillips EH. Abdominal wall hernias. Springer Verlag, New York, 2001 pp 500-503.

12. Langer C, Schaper A, Liersch T, Kulle B, Flosmann M, Füzesi L, et al. Prognosis factors in incisional hernia surgery: 25 years of experience. Hernia 2005; 9: 16-21.

13. Luijendijk RV, Hop WC, van den Tol MP, de Lange DC, Braaksma MM, IJzermans JN, et al. A comparison of suture repair with mesh repair for incisional hernias. New Engl J Med 2000; 343: 392-398.

14. Schumpelick V. Hernien. Georg Thieme Verlag, Stuttgart 2000; pp 266-302.

15. Morris-Stifff GH, Huges LE. The outcomes of nonabsorbable mesh placed within the abdominal cavity: literature review and clinical experience. J Am Coll Surg 1998; 186: 352-367.

16. Paul A, Korenkov M, Peters S, Köhler L, Fischer S, Troidl H. Unacceptable results of Mayo procedure for repair of abdominal incisional hernias. Eur J Surg 1998; 164: 361-367.

17. Sauerland S, Schmedt CG, Lein S, Bittner R. Primary incisional hernia repair with or without polipropilene mesh: a report on 348 patients with 5 year follow-up. Langenbecks Arch Surg 2005; 390: 408-412.

18. Welti H, Eudel F. Un procédé de cur readical des éventrationes postoperatoires par auto-étalement muscles grand droits, apreés incisión du feuillet anterior de leurs gaines. Mem Acad Chir 1941 ; 28: 781-798.

19. Israelsson LA, Smedberg S, Montgomery A, Nordin P, Spangen L. Incisional hernia repair in Sweden. 2002 Hernia 2006; 10: 258-261.

20. Korenkov M, Sauerland S, Arndt M, Bograd L, Neugebauer FAM, Troidl H. Randomized clinical trial of suture repair, polypropylene mesh or autodermal hernioplasty for incisional hernia. Br J Surg 2002; 89: 50-56. 